\title{
All Optical Packet Switches Based On Space Switch Array for the Transmission of Higher Data Rate Using NRZand RZ Modulation
}

\author{
Divya $\mathrm{T} \mathrm{S}^{1}$, Avani Nath $\mathrm{N} \mathrm{J}^{2}$ \\ ${ }^{1} P G$ scholar, ${ }^{2}$ Assistant Professor Dept. of electronics and communication engineering \\ TKM institute of technology, Kerala, India-691505.
}

\begin{abstract}
The explosive growth of the Internet traffic requires the design of next generation switching systems accommodating huge data-centric traffic at the packet level. All-optical packet switching (OPS) with greater flexibility, granularity and switching capacity is able to meet the complexity of traffic. An all-optical fiber-optic switching device maintains the signal as light, from input to output. Semiconductor optical amplifiers (SOAs) are widely studied as nonlinear elements for high bit rate all-optical switching applications, such as wavelength conversion and regeneration. All optical switches incorporating SOAs have peculiarities like small size, low switching energy requirement and high non linearity. Here simulation of a $4 x 4$ optical packet space switch array based on Optically Amplified Suppressed Interference Switches (OASIS) with different data rate has been performed. The OptiSystem version-12 simulator is used to model the structure, it is also used to assess the behavior and performance of this switch array. The simulation of $4 \times 4$ space switch array with different bit rate and for different modulation format is done which gives the BER within the acceptable range. Implications of cascadability of this switch array will be analysed which improves the quality and capacity of the existing networks
\end{abstract}

Key words: Optical Switch, Optical splitter, Q-Factor, BER, Turbo switch

\section{Introduction}

Communication is the process of transmission of information from one point to another through a guided or unguided medium. Today's tremendous data transmission demand can't be fulfilled by the electronic transmissions. To fulfill such huge demands optical transmission technology is required. Such systems can send 10 to 100 wavelengths per fiber with each wavelength modulated at $10 \mathrm{~Gb} / \mathrm{s}$ or more.

In order to exploit the tremendous capacity provided by optical fiber, the switching functions must be implemented in optical domain. [1] High-speed optical switching elements will play a crucial role in future highly flexible, all-optical network in the effort of moving the packet-switching functions to the optical layer. To support packet data routing, optical packet-switching elements having high extinction ratios, low polarization-dependent loss, low loss, and high-scalability are highly desirable.

In electro optic switches at every switching node, optical signals are converted to electrical form (O/E conversion), buffered electronically, and subsequently forwarded to their next hop after being converted to optical form again (E/O conversion). As data traffic starts to dominate the communication networks, the traffic even on the long-haul network becomes more data oriented (i.e., less predictable). In the long term, Optical Packet Switching (OPS) could become a viable candidate because of its high-speed, fine granularity switching, flexibility, and its ability to use the resources economically.

As the network capacity increases, electronic switching nodes seem unable to keep up. Apart from that, electronic equipment is strongly dependent on the data rate and protocol and thus, any system upgrade results in the addition and/or replacement of electronic switching equipment. The main attraction of optical switching is that it enables routing of optical data signals without the need for conversion to electrical signals and, therefore, is independent of data rate and data protocol.

The transfer of electronic switching to optic switching results in reduction of the network equipment and increase in switching speed and thus improves the network throughput. In addition, the elimination of $\mathrm{E} / \mathrm{O}$ and O/E conversions will result in a major decrease in the overall system cost, since the equipment associated with these conversions represents the lion's share of cost in today's networks

An optical switch adds manageability to a DWDM node that could potentially grow to hundreds of channels. An O-O-O switch holds the promise of managing those light signals without converting the signals to electrical and then back again [2]. This is especially attractive to those carriers operating large offices where up to $80 \%$ of the traffic is expected to pass through the office on its way to locations around the globe. 


\section{System Architecture}

\section{A. Data flow in a Space Switch Array}

Space switches and wavelength routers are basic components of an optical switch. Data flow in a space switch array is shown in Figure 1.A space switch simply switches signals from each input to one of the outputs.[3] There are several ways to implement a space switch, but a semiconductor optical amplifier (SOA) technology seems to be the best alternative.

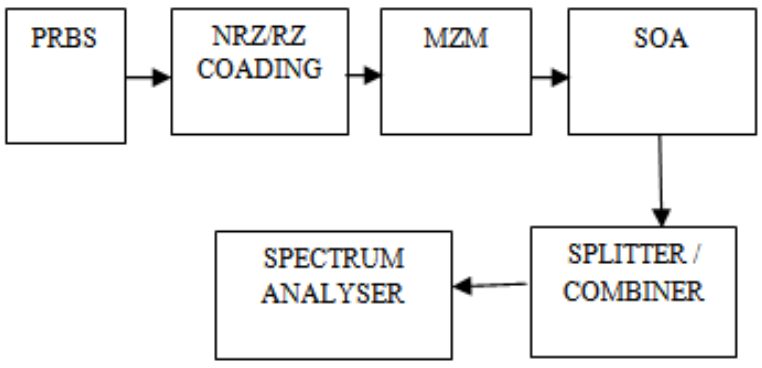

Figure 1: Data flow in a Space Switch Array

Data to be transmitted is generated using p-n sequence generator is given to $\mathrm{CW}$ laser operating at $1550 \mathrm{~nm}$.Then the signal is given to SOA which acts as a switch according to the junction current. The selector or combiner is used for selecting the path and the data reaches at the output port. Routing is Routing is realised by applying currents to turn on the relevant gates along the route. In the absence of a current, the signal is suppressed to a very low level (about $-50 \mathrm{~dB}$ ), which effectively reduces crosstalk.

\section{B. Block Diagram Of $4 \times 4$ Space Switch Array}

The 4x4 switch architecture shown in Figure 1 considered is a strictly non blocking broadcast and select architecture composed of $\mathrm{N}$ input 1: $\mathrm{N}$ splitters interconnected with $\mathrm{N}$ output. This space switch architecture based on $4 \times 1$ SOA building block sub-elements is modular, flexible and thus can be easily extended and, of course, the same building blocks are re-usable in another switch architecture. Data to be transmitted is generated using p-n sequence generator is given to $\mathrm{CW}$ laser operating at $1550 \mathrm{~nm}$.

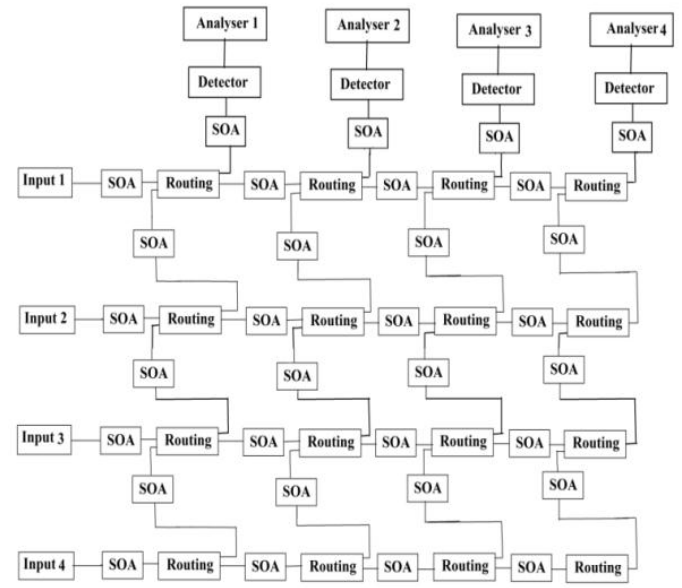

Figure 2: Block Diagram of $4 \times 4$ space switch array

\section{Simulation Of $4 \times 4$ Space Switch Array}

In this work, Simulations of a 4 x 4 optical packet space switch array by using both NRZ and RZ modulation format for different data rate is performed.

The simulation diagram for $4 \mathrm{X} 4$ space switch array is shown in Figure.3. The performance of $4 \mathrm{X} 4$ space switch array was analyzed using an input data rate of $10 \mathrm{Gbps}$ and the Eye Diagrams were observed at four output nodes. Eye Diagrams thus obtained were studied and the corresponding output parameters were noted. 


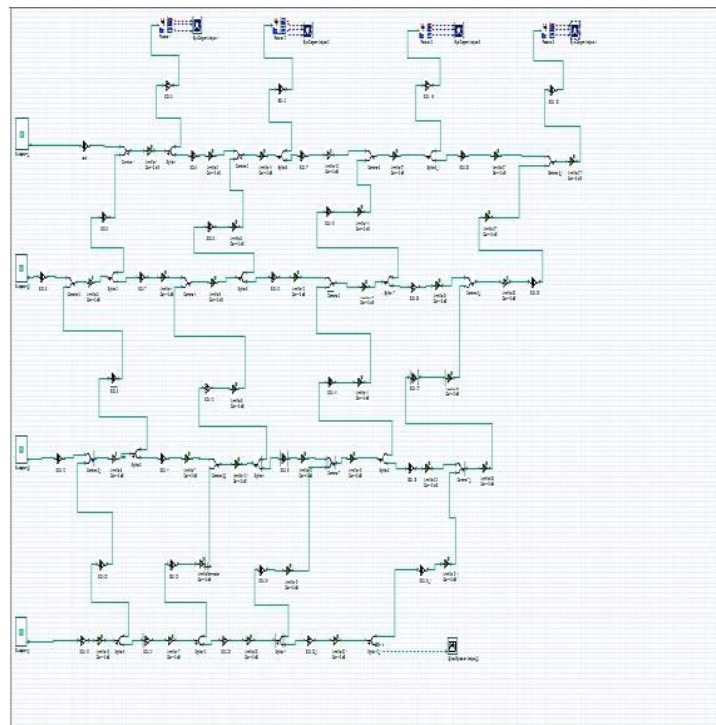

Figure 3: Simulation layout of $4 \times 4$ space switch

After having successfully achieved optimum BER and quality this switch array is used to design optical communication systems. Simulation can be done to determine their performance given various component parameters to guarantee the highest possible accuracy and real-world results. OptiSystem represents an optical communication system as an interconnected set of blocks, with each block representing a component or subsystem in the communication system. As physical signals are passed between components in a real world communication system, "signal" data is passed between component models in the OptiSystem simulation. Each block is simulated independently using the parameters specified by the user for that block and the signal information passed into it from other blocks. This is known as a block-oriented simulation methodology.

These blocks are graphically represented as icons in OptiSystem. Internally they are represented as data structures and sophisticated numerical algorithms.

Table 1results Obtained From The Simulation 4x4 Space Switch Array With 10Gbps

\begin{tabular}{|c|c|c|c|c|}
\hline & \multicolumn{2}{|c|}{ With NRZ coding } & \multicolumn{2}{c|}{ With RZ coding } \\
\hline Analyser & BER & Q value & BER & Q value \\
\hline Analyser1 & $1.843 \times 10^{-3}$ & 2.8683 & $5.5 \times 10^{-4}$ & 3.25716 \\
\hline Analyser2 & $1.6732 \times 10^{-5}$ & 4.1240 & $7.0485 \times 10^{-6}$ & 4.31916 \\
\hline Analyser3 & $1.9006 \times 10^{-12}$ & 6.9365 & $6.9029 \times 10^{-13}$ & 7.07926 \\
\hline Analyser4 & $1.0898 \times 10^{-13}$ & 7.3233 & $1.67 \times 10^{-15}$ & 7.86444 \\
\hline
\end{tabular}

Table 2 Results Obtained From The Simulation 4x4 Space Switch Array With 40Gbps

\begin{tabular}{|l|c|c|c|c|}
\hline & With NRZ coding & \multicolumn{2}{c|}{ With RZ coding } \\
\hline Analyser & BER & $\begin{array}{c}\text { Q } \\
\text { value }\end{array}$ & BER & Q value \\
\hline Analyser1 & $6.704 \times 10^{-3}$ & 2.4009 & $4.768 \times 10^{-3}$ & 2.5348 \\
\hline Analyser2 & $1.4648 \times 10^{-4}$ & 3.5752 & $8.2628 \times 10^{-5}$ & 3.7310 \\
\hline Analyser3 & $2.9075 \times 10^{-7}$ & 4.9853 & $7.42108 \times 10^{-7}$ & 4.8107 \\
\hline Analyser4 & $2.5358 \times 10^{-8}$ & 5.4315 & $2.45601 \times 10^{-7}$ & 5.0152 \\
\hline
\end{tabular}

The simulation uses Non Return to Zero (NRZ) and Return to Zero (RZ) PRBS signals at the data rate of $10 \mathrm{~Gb} / \mathrm{s}$ and $40 \mathrm{~Gb} / \mathrm{s}$. The data stream modulates a laser with $\mathrm{CW}$ power of $0.1 \mathrm{~mW}$ at a central frequency of $1550 \mathrm{~nm}$.

Boosters were used in the simulation of $4 \times 4$ space switch arrays and the Eye Diagrams of the output signal at all the four scopes were analyzed for the data rate of $10 \mathrm{Gbps}$ and $40 \mathrm{~Gb} / \mathrm{s}$. 

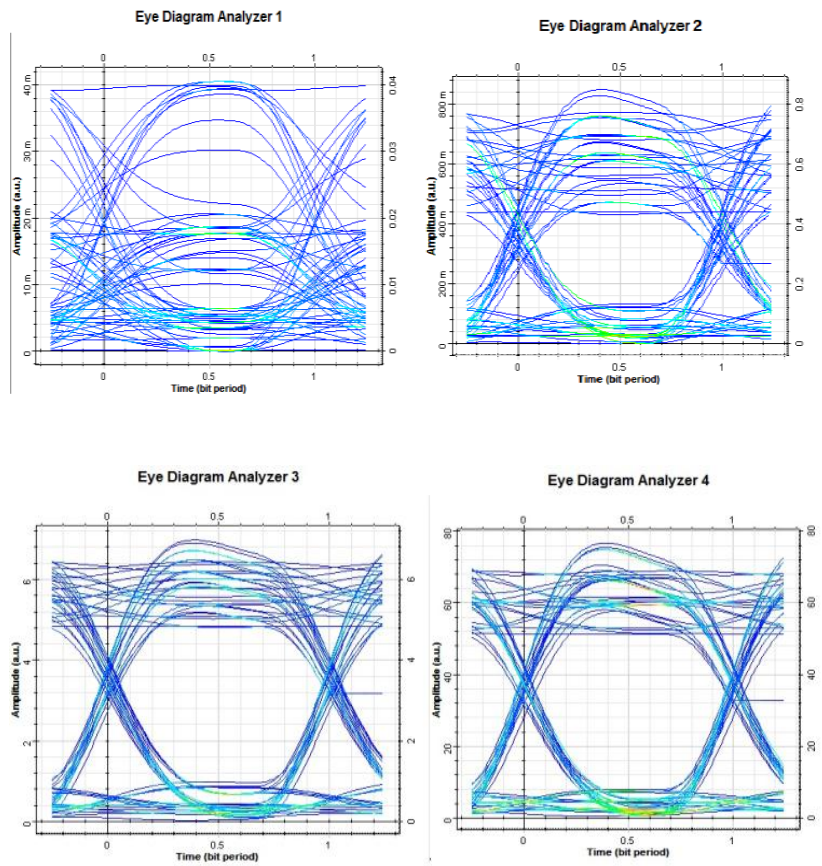

Figure 4: Eye diagram obtained for the simulation of $4 \times 4$ switch array using $10 \mathrm{~Gb} / \mathrm{s}$ using NRZ coding
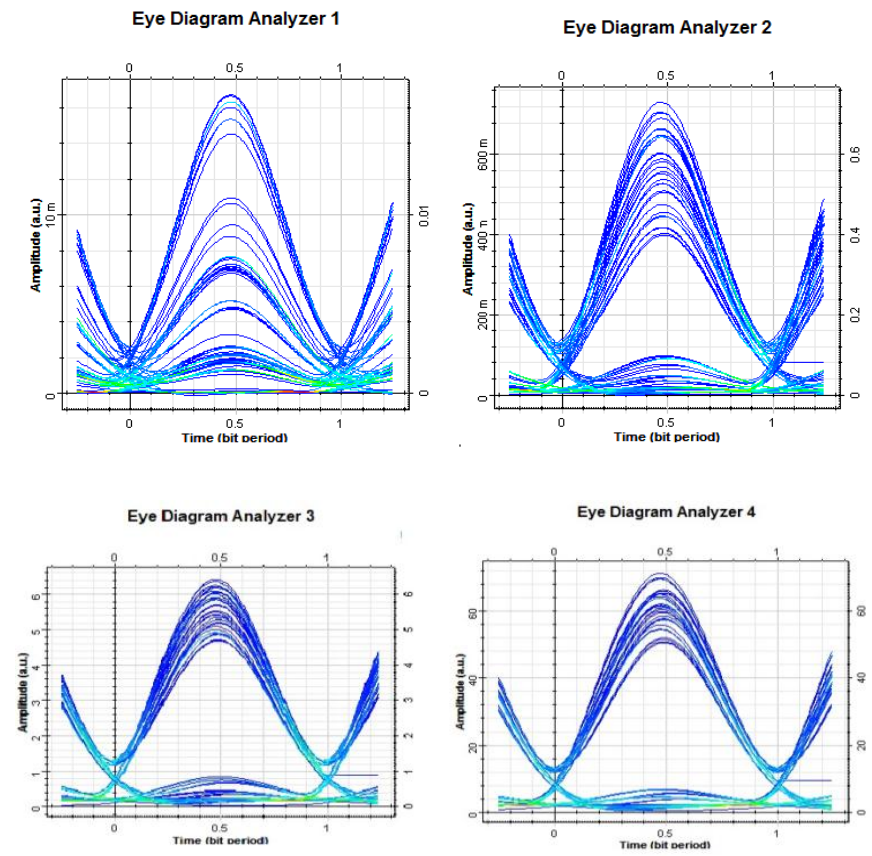

Figure 5: Eye diagram obtained for the simulation of $4 \mathrm{x} 4$ switch array using $10 \mathrm{~Gb} / \mathrm{s}$ using RZ coding
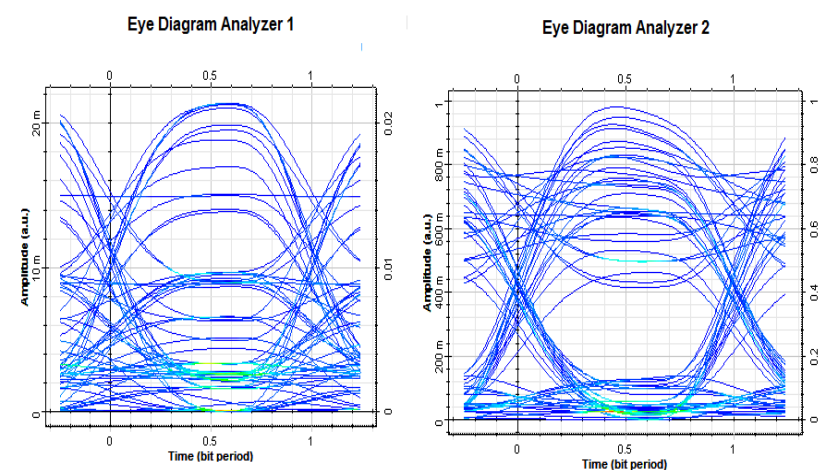
Eye Diagram Analyzer 3

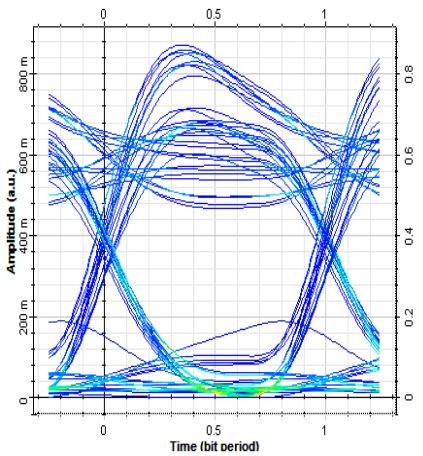

Eye Diagram Analyzer 4

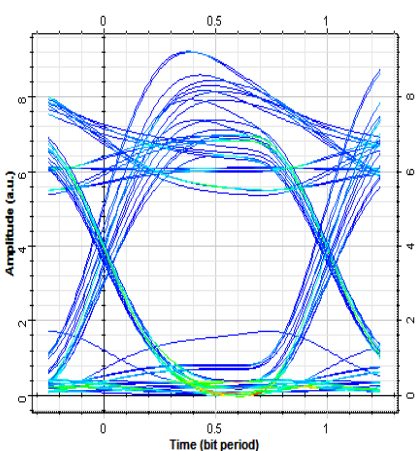

Figure 6: Eye diagram obtained for the simulation of $4 \mathrm{x} 4$ switch array using $40 \mathrm{~Gb} / \mathrm{s}$ using $\mathrm{N}$ RZ coding

Eye Diagram Analyzer 1

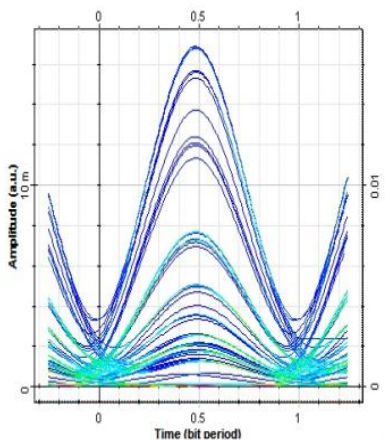

Eye Diagram Analyzer 3

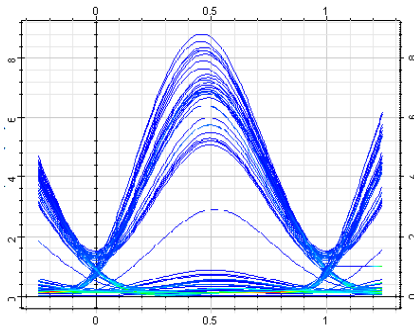

Eye Diagram Analyzer 2

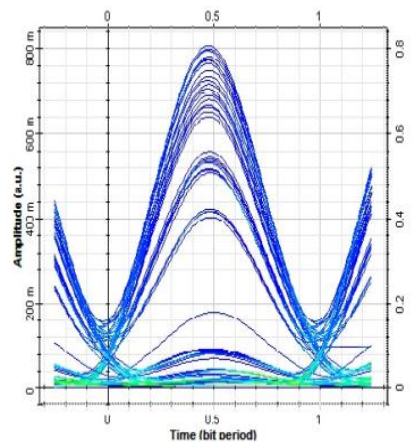

Eye Diagram Analyzer 4

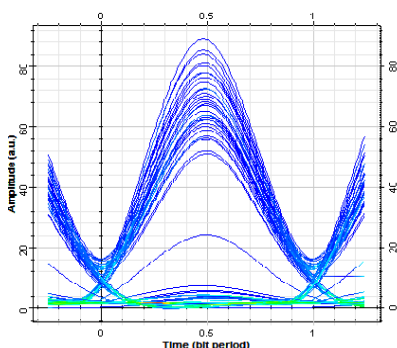

Figure 7: Eye diagram obtained for the simulation of $4 \times 4$ switch array using $40 \mathrm{~Gb} / \mathrm{s}$ using RZ coding

Figure 4, Figure 5, Figure 6 and Figure 7 show the Eye Diagrams for the output signal at scope1, scope2, scope 3 and scope 4 with NRZ and RZ coding for $10 \mathrm{~Gb} / \mathrm{s} \mathrm{ND} 40 \mathrm{~Gb} / \mathrm{s}$.

The simulation of a $4 \times 4$ space switch array with a data rate of $10 \mathrm{~Gb} / \mathrm{s}$ for NRZ data format gives $\mathrm{Q}$ value ranging from 2.8683 to 7.32332 and a Bit Error Rate (BER) ranging from $1.843 \times 10^{-3}$ to $1.08981 \times 10^{-13}$. The simulation of a $4 \times 4$ space switch array with a data rate of $10 \mathrm{~Gb} / \mathrm{s}$ for RZ data format gives $\mathrm{Q}$ value ranging from 3.25716 to 7.864447 and a Bit Error Rate (BER) ranging from $5.5 \times 10^{-4}$ to $1.67 \times 10^{-15}$. The simulation of a $4 \times 4$ space switch array with a data rate of $40 \mathrm{~Gb} / \mathrm{s}$ for NRZ data format gives $\mathrm{Q}$ value ranging from 2.40097 to 5.43153 and a Bit Error Rate (BER) ranging from $6.704 \times 10^{-3}$ to $2.5358 \times 10^{-8}$. The simulation of a $4 \times 4$ space switch array with a data rate of $40 \mathrm{~Gb} / \mathrm{s}$ for $\mathrm{RZ}$ data format gives $\mathrm{Q}$ value ranging from 2.53481 to 5.01522and a Bit Error Rate (BER) ranging from $4.768 \times 10^{-3}$ to $2.45601 \times 10^{-7}$.

\section{Conclusion}

Physical layer switches are vital to supporting the increase in speed and the efficiency of the network growth in key application areas such as test automation, network monitoring, cloud networking, and Cyber Security. Electrical switches can meet many of these needs for networks that operate at speeds up to $10 \mathrm{Gbps}$, All-optical switches are the only choice for networks that go beyond the $10 \mathrm{Gbps}$ data rate. Here simulations 
using OptiSystem investigating the behavior and performance of 4 x 4 optical packet switches were described. The results of the study on a single switch unit as well as a cascaded switch network were then discussed.

The $4 \times 4$ switch array performs satisfactorily for a small number of nodes. When data trains are used to switch these devices, the slow SOA lifetime leads to patterning in the gain and phase response of the SOA. In order to prevent such patterning, a faster response speed is generally required. Hence modification of the current switching elements needs to be done. One such arrangement, known as Turbo-Switch which uses two SOAs and a filter as the basic switching element can be implemented in future. The recovery time of a Turbo-Switch is less as compared to SOA and hence higher switching speeds can also be achieved.

\section{References}

[1]. Lochan Jolly, Rashmita Kumari Mohapatra " Optical Packet Switching using Space Switch Arrays" International Journal of Computer Applications (097- 8887) International Conference and Workshop on Emerging Trends in Technology 2013

[2]. G.I. Papadimitriou, C. Papazoglou, A.S. Pomportsis "Optical switching:switch fabrics, techniques, and architectures" Journal of Lightwave Technology. Vol 21 03/2003; 21(2):384- 405

[3]. Ravinder Yadav, Rinkle Rani Aggarwal "Survey and Comparison of Optical Switch Fabrication Techniques and Architectures" Journal of computing, Vol 2, Issue 4, April 2010

[4]. Po Dong, Shirong Liao, Hong Liang, Roshanak Shafiiha "Submilliwatt, ultrafast and broadband electro-optic silicon switches" Optics Express, Vol. 18, Issue 24, pp. 25225-25231,2010

[5]. S Ponmalar and S Sundaravadivelu " Design of ultra fast $2 \times 2$ electro-optic polymer waveguide switch for protection in intelligent optical networks" Journal of Scientific \& Industrial Research Vol. 70, January 2010

[6]. Yuya Shoji, Kenji Kintaka, Satoshi Suda, Hitoshi Kawashima "Low-crosstalk $2 \times 2$ thermo-optic switch with silicon wire waveguides" Optics Express, Vol. 18, Issue 9, pp. 9071-9075 (2010)

[7]. Dan Owen Harris and A. VanderLugt "Acousto-optic photonic switch" Optics Letters, Vol. 14, Issue 21, pp. 1177-1179 (1989)

[8]. W. Yuan, S. Kim, G. Sadowy, C. Zhang, C. Wang, W.H. Fetterman "Polymeric electro-optic digital optical switches with low switching voltage" Electronics letters Vol. 40,5th February 2004

[9]. Le Nguyen Binh and Zsofia Csematony "Double-Sideband Carrier Suppressed RZ and NRZ Modulation Formats for Ultra-high Capacity 40 Gb/s DWDM Optical Communications Systems" MECSE-23-2003

[10]. Rekha Mehra,Shikha Jaiswal, Hemant Kumar Dixit "Optical computing with semiconductor optical amplifiers" Optical Engineering 51(8), August 2012 